\title{
Multi-Hub Network Configurations - A Temporary or Permanent Outcome of Airline Consolidation?
}

\author{
JAN-CHRISTOPH DÜDDEN * \\ WHU Graduate School of Management
}

\begin{abstract}
Ongoing consolidation in the European airline industry poses a threat to second-tier hub cities in terms of losing hub status and long haul routes. As airlines merged in the USA, it was often more efficient to close a duplicating hub and concentrate resources on the stronger one. This paper relies on a formal model to explore discrete scenarios for non-hub intercontinental connections. The results of the analysis show that a hub and spoke airline in a competitive environment can rationally preserve or initiate non-hub long haul routes, even going to the length of maintaining or building up an ancillary hub.
\end{abstract}

\section{Introduction}

Industry observers have been anticipating consolidation in the European airline industry for a long time. Many agree that the flag carrier system in Europe has created a fragmented industry structure with too many airlines operating too much inefficient capacity out of too many hubs. Prior to 2003, the only notable move towards consolidation was the demise of Sabena - the former Belgian national flag carrier - in the wake of the September, $11^{\text {th }}$ terrorist attacks in 2001. KLM had been involved in two unsuccessful merger attempts (KLM/British Airways and KLM/Alitalia) before finally merging with Air France in 2003. Approval by the EU regulators sparked expectations for a merger wave in Europe, but despite a multitude of press speculations with varying validity, only Lufthansa has followed suit by acquiring Swiss in early 2005.

Today, Scandinavian Airlines, Austrian Airlines, Alitalia and Iberia are the remaining second tier legacy carriers in Europe. The latter has obtained a cross equity stake with Oneworld-Alliance partner British Airways, while SAS and AUA are both perceived to work very close with Star Alliance partner Lufthansa, but with no merger intentions on the cards. Last but not least, Alitalia has been associated with both Lufthansa and SkyTeam partner AF/KLM. Clearly, the European market offers further potential, but the early stage of consolidation does not help much with predictions on how the future landscape will look like.

\footnotetext{
* Am Ritterkeller 3, D-61118 Bad Vilbel, Germany. Tel.: +49 (151) 1 9557559. E-Mail: jduedden@,hudsonadvisors.de
} 
On the other side of the Atlantic Ocean, the US market has already undergone several merger waves. Of the over 50 US carriers which surfaced after deregulation, nearly all have failed or been consolidated into the six major US network carriers. In retrospective, closing down or reducing activity at smaller hubs and reallocating flights to the larger hub has been a common outcome of US airline mergers ${ }^{1}$, resulting in a greater concentration on a smaller number of larger hubs. With continuing consolidation in Europe, the same destiny could have hit the second-tier hubs like Zurich, Amsterdam, Milano or Vienna.

Despite this trend, the major US carriers still operate multiple hubs ${ }^{2}$, but no two hubs of the same carrier are geographically concentrated. For instance, United's hubs in Chicago, Denver, Los Angeles, San Francisco and Washington are well spread throughout the US. This allows the US network carriers to serve different geographical flows with each hub.

A similar constellation could be imagined in a British Airways/Iberia merger. Both carriers have developed a geographical specialization based on the locational advantages and disadvantages of their respective hub locations. British Airways has focused on transatlantic traffic to North America, while Iberia is very present on the South American long haul market. Due to this rather low route overlap and the distance between both hub cities, a merger would not necessarily imply consequences for Madrid as a redundant hub location.

These arguments do not necessarily apply to the AF/KLM and Lufthansa/Swiss groups, where the close proximity of the formerly competing hubs eliminates any distinguishing locational advantages for specific traffic flows. Concerning AF/KLM, we have an artificial barrier to hub consolidation in place, as Air France gave assurances to the Dutch State specifying that KLM's hub in Amsterdam is not dismantled for a duration of eight years as part of the merger agreement. This restriction aside, a logical step for Air France/KLM would have been to concentrate resources on the stronger hub, and close the duplicating weaker one. By keeping both hubs in operation, the merged company forgoes the most obvious synergies for a hub and spoke carrier: to maximise economics of density in a single hub location. Market observers remain anxious if this will prevail beyond the expiration of the clause in 2011.

The Lufthansa/Swiss Group has not indicated any planned hub downsizing activities either, even though the merger with Swiss has added Zurich as a third hub less than an hour flying time away from its hubs in Frankfurt and Munich. ${ }^{3}$ The merger agreement sees the long term development of the Zurich hub on an equitable basis to Frankfurt and Munich, and even envisions the addition of long haul capacity in the short term.

So are there key differences between the European and the US market? Judging from the hopes of all market participants that consolidation would be the remedy for a still weak European airline industry, one must wonder what the solution will be, if not coming from capacity reductions and hub consolidation. But while the AF/KLM group is legally bound

\footnotetext{
${ }^{1}$ American Airlines closed down the Raleigh-Durham and St.Louis hubs after the acquisitions of Eastern Airlines TWA. Delta reduced hub operations in Los Angeles following the purchase of Western Airlines. Similarly, US Airways closed down the Baltimore hub when merging with Pacific Southwest Airlines. Other hub operations were frequently discontinued in times of financial distress.

${ }^{2}$ United Airlines: 5; Delta: 3, American Airlines: 4, US Airways: 2; Continental: 3; Northwest: 3

${ }^{3}$ In the past years, Munich had been developed as a secondary hub to cope with the capacity constraints of Frankfurt/Main Airport that were limiting growth of Lufthansa's network.
} 
to operate both hubs, Lufthansa seems to deliberately pursue a multi-hub strategy, selling it as a competitive advantage and positive differentiation criterion.

In the scope of this paper, we want to evaluate demand considerations that can lead to deviations from the single-hub as the profit maximising network form. Section 2 discusses the existing work on network configurations and gives some factual evidence. In section 3 , we develop a formal model of two competing hub and spoke carriers confronted with the decision to establish a non-hub route. Section 4 discusses the issues raised by the model and Section 5 concludes.

\section{Multi-Hub network configurations}

Abundant literature exists on air transportation networks and the cost and demand conditions of an airline that are the main determinants of network choice. However, much of this work is confined to the two polar cases of point-to-point and hub and spoke network configurations. A further common restriction is a three city setup, where a hub and spoke configuration implies the existence of a solitary hub. In settings with networks of arbitrary size and structure, most studies have found no evidence for multi-hub networks existing as a result of cost-minimizing behaviour under symmetric cost functions. ${ }^{4}$ Looking beyond simple cost issues in investigating network choice yields possible demand related aspects, for example, consumer choice or competition. To our knowledge, none of these have been examined in detail as a cause to partly deviate from a hub and spoke network.

In competitive markets, Oum et al. (1995) regard hubbing as offering a strategic advantage. Lower marginal costs achieved through economies of density induce higher outputs, hence reducing the profitability of entry of other carriers. However, it remains unclear how multiple hubs within a network could amplify the strategic gain as opposed to a singular hub, since the key driver - density economies - decreases with each additional hub. Viable explanations could be a cessation of density economies at high hubbing levels ${ }^{5}$ or an excess of demand that cannot be met at a single hub location, for example, due to airport capacity constraints. In the latter case, a secondary hub can capture the demand spill; in the former, a second hub can extract further strategic advantages from hubbing.

In this paper, we want to consider the impact of demand considerations and if these can lead to deviations from the single-hub as the profit maximising network form. Existing work has shown that hub and spoke networks are more likely to be optimal when the value of flight frequency is high and the disutility of longer travel times and connecting are low. ${ }^{6}$ Assuming that singular markets exist, where consumers highly value direct travel, this could explain dedicated non-hub direct services. The spatial configuration would then resemble a multi-hub network, even if no transferring is taking place.

We factor out airport capacity constraints and geographical specialization from our analysis as we deem both as viable reasons for the existence of multi hub networks. We

\footnotetext{
${ }^{4}$ See Hendricks et al. (1995) for a framework with symmetric demand and Wojahn (2001) for the case of asymmetric demand. The assumption of symmetric cost functions implies a spatially symmetric or spaceless world.

${ }^{5}$ Density economies are primarily driven by aircraft size, which cannot take infinite numbers. Similarly, strategic marketing effects of additional frequencies are rather low at already high frequency levels (Wojahn, 2001).

${ }^{6}$ Compare, for example, Berechman and Shy (1996) and Brueckner and Zhang (1999).
} 
confine our analysis to long haul travel, as we more readily perceive the need for hub and spoke networks. ${ }^{7}$ However, the results can be easily expanded to general hub and spoke networks. We consider short haul travel as less relevant, as the secondary European hub cities all represent major cultural and economic centres of their respective countries. Hence, the number of short haul operations may shrink in terms of frequency in the aftermath of a merger, but the number of destinations is expected to remain on a similar level. Furthermore, the emergence of low cost carriers represents factual evidence that short haul markets can efficiently and profitably be connected through point-to-point networks. Whilst in the past, hub and spoke carriers still had the advantages of network quality over low cost carriers, this competitive lead is starting to disappear as low cost carriers are stocking up frequencies and their networks are becoming ever denser.

Our work builds on the notion that premium demand, given a sufficient frequency, will prefer a direct flight to the corresponding transfer product. This thought is supported by available information on consumer behaviour. Figure 1 shows the correlation between the percentage of business class travellers opting for the direct flight and the quantity of daily frequencies. The sample includes 270 markets with a minimum average of half a flight per day throughout 2002.

Percentage of direct travelling high yield demand compared to frequency

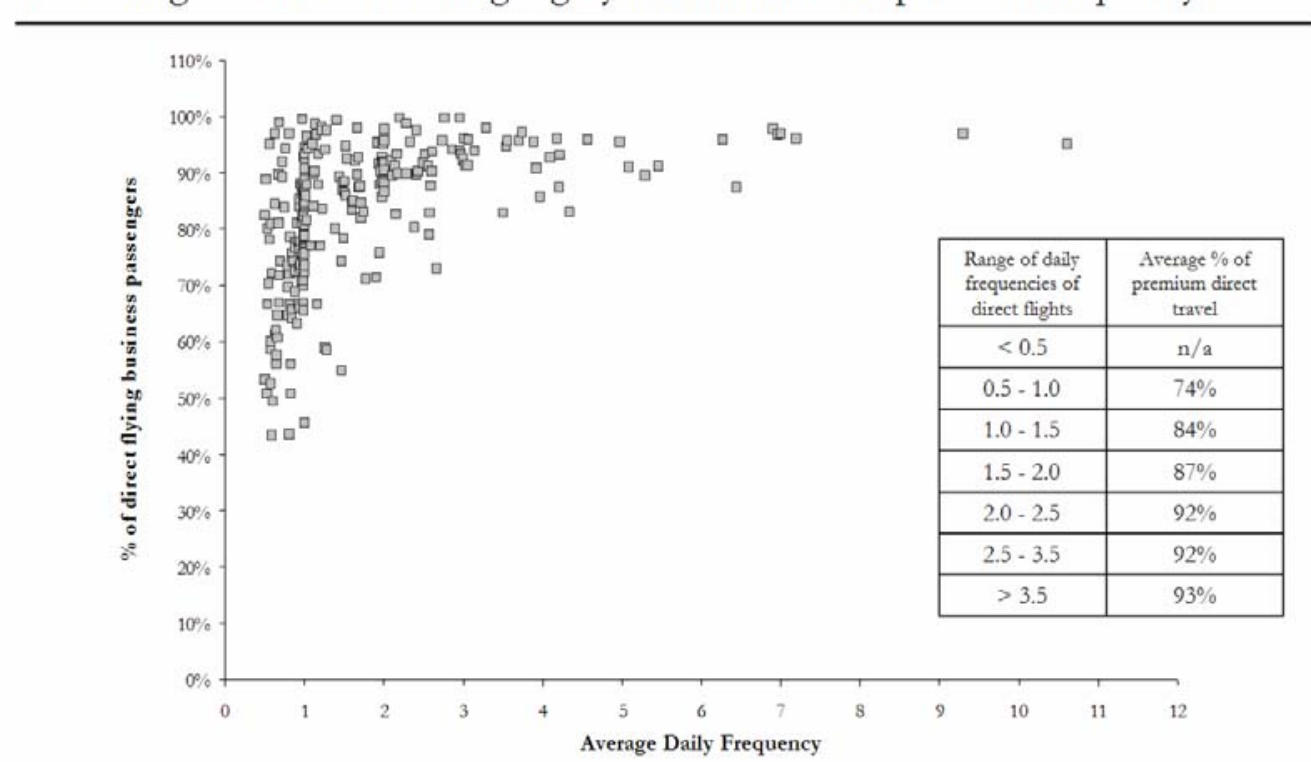

\section{Figure 1}

With rising frequencies, fewer passengers rely on the vast amount of available transfer products. As the prices of transfer connections are typically below those of direct offerings,

\footnotetext{
${ }^{7}$ Considering recent demand levels, a linear network configuration could establish 100 routes that connect 19 European cities with 50 intercontinental destinations on a regular basis. Served demand would be approximately 27 million passengers per annum. Currently, we have 89 million long haul passengers per year, which implies that 62 million passengers would be deprived of long haul travel. Or, in other words: $69 \%$ of long haul passengers are dependent on the existence of hub and spoke networks and the 10,000 possible indirect connections they provide. In our view, 62 million potential customers represent a strong case for the future existence of hub and spoke networks. In comparison, reservation system data shows that $84 \%$ of intra-European travel is non-stop. As internet and call-center bookings of Low Cost Carriers are not included, the real figure is probably even higher.
} 
this preference cannot be explained by consumers' price sensitivity. Low yield demand displays a similar relation, albeit at a much lower level of only $66 \%$ vs. the average of $82 \%$ for high yield travel. Further, deeply discounted prices for economy travel often do not include a premium for direct travel, so we cannot necessarily say that the decision to fly directly is independent of price.

\section{The model}

The model has $n$ hub and spoke carriers which are in competition with each other for long haul travel demand from a spoke city $S$ to destination $D, q_{S D}$. For the model description, we will relate to the duopoly case and denote the airlines $A$ and $B$ (compare Figure 2). For simplification, prices and demand are exogenously given and apply to all market participants in an equal way. ${ }^{8}$

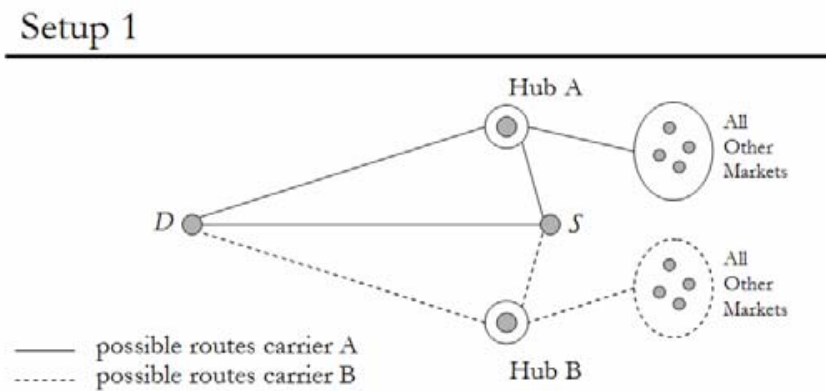

Figure 2

We use a linear cost function $M C(q)=1-\phi q$ to determine the costs of servicing the long haul routes, which is influenced by a returns to density parameter $\phi$. The cost of feeding a passenger into the hub from the spoke city is assumed to be independent of the returns to density parameter and is described by $F$. $^{9}$

Passengers' preference for non-stop flights is modelled by assuming that a certain percentage $(\lambda)$ will rearrange their travel itineraries to take the direct connection, if one is available. The residual demand evenly distributes itself among the transfer connections via hubs.

We further define the feasible parameter space by the inequalities that result from the requirements of non-negative marginal costs (A1) and quantities (A2).
(A1) $M C(q) \geq 0$
(A2) $q_{i} \geq 0$

\footnotetext{
${ }^{8}$ In the appendix, we show why we do not deem this rather severe restriction of exogenously setting prices and demand to be crucial for the results.
} 
The level of realizable density economics is further determined by the size of other spoke markets that are routed via the hub and is described by $q_{a o m} . q_{a o m}$ represents demand from all other spoke markets to destination $D$ that can only be served by hub and spoke networks and is equal for both hub and spoke carriers.

We derive the profit function for demand transported via the hub given by

$$
\Pi_{H S C}\left(q_{S D}\right)=\left(p^{*} q_{S D}\right)-q_{S D}\left(F+M C\left(q_{S D}+q_{a o m}\right)\right)
$$

The carriers are confronted with two possible network configurations. A pure hub and spoke network (PHS) represents a decision against a non-hub offering and solely relies on the transfer product through the respective hub: alternatively, the carrier can employ a bypassed hub network $(B H N)$, which is a direct long haul product on the non-hub route with the additional option of travelling via the hub.

Assumption 3 implies that offering non-stop capacity is more costly than offering transfer capacity, which makes a pure hub and spoke network the cost optimal network form. ${ }^{10}$

(A3) $F<\phi^{*} Q_{\text {AOM }}$

To simplify, we will start our analysis by only allowing one of the hub and spoke carriers to make a network decision regarding the spoke market. The competing carrier $B$ is restricted to option PHS, only allowing service of the spoke market via transfer connections. Although seeming rather restrictive, the underlying rationality is the continuing existence of bilateral traffic agreements in Europe. In the event of consolidation through mergers, traffic rights will only fall to one carrier, which can then decide on how and if to continue the flight offering in the newly acquired hub city.

Equations (2) and (3) give the profit functions of carrier $A$ based on its network decision, when the competing carrier $B$ is restricted to a pure hub and spoke network configuration:

$$
\begin{aligned}
& \Pi_{P H S(P H S)}=\Pi_{H S C}\left(\frac{Q_{S D}}{n}\right) \\
& \Pi_{B H N(P H S)}=\left(p * \lambda Q_{S D}\right)-\left(\lambda Q_{S D} * M C\left(\lambda Q_{S D}\right)\right)+\Pi_{H S C}\left(\frac{(1-\lambda) Q_{S D}}{n}\right)
\end{aligned}
$$

Then we have the following result.

\footnotetext{
${ }^{9}$ Density economics also exist for short haul travel, but we regard these to be negligible for our case. The assumption additionally remains defensible, as the hub and spoke carrier could outsource feeder services to another partner (regional airline, train) which would be paid on a per passenger basis.

${ }^{10}$ This assumption is required to factor out the possible outcome, of the bypassed hub network being the profit maximising network form due to lower costs.
} 
Proposition 1: Assume that offering a non-hub direct connection attracts a share of demand, then it is rational for a carrier to provide such an offering, when the revenue increase exceeds the higher costs of operation.

When carrier $A$ establishes a direct connection, it not only harms the competitor by diverging demand, but thereby increases its own revenues. Splitting demand among the direct and the transfer connection meanwhile leads to a loss in density economics that simultaneously increases costs. When the additional revenues $\lambda * Q_{S D} * \frac{(n-1)}{n}$ exceed the latter, then it is profit maximising for carrier $A$ 's network to operate the direct flight. The revenue increase originates at the route level, while the cost increase takes place at the network level. Beyond revenues remain unaffected, as carrier $A$ continues to operate the spoke route to the hub.

These results can be expanded to give some insight into the rationality of multi-hub networks.

Proposition 2: Assume that offering a direct non-hub connection increases the profitability of an airline's network despite city pair demand being insufficient for the profitable operation of aircraft, then the airline adopts a multi-hubbing network.

Our model assumes capacity to be continuous. In a setting, where air transportation only becomes economically feasible with a minimum size of aircraft that exceeds available market demand, it can be rational to route other spoke market passengers with a corresponding destination through the origin city to achieve the minimum demand threshold. The city then assumes the role of a secondary hub. Albeit not belonging to the category of cost-minimizing networks, a multi-hub network can be profit maximising, if the improvements on the revenue side can offset the loss of density economies in the primary hub.

To demonstrate this, we add another spoke market with demand $Q_{2}$. $Q_{\text {Min }}$ reflects the minimum demand required for the profitable operation of aircraft. Figure 3 shows the new setup with the added spoke market.

\section{Setup 2}

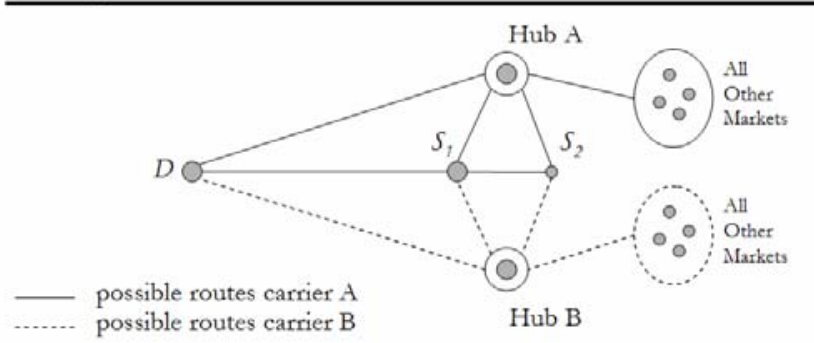

Figure 3

This gives us the profit function for a pure hub and spoke network and a multi hub network configuration $(M H N)$. 
(4)

$$
\Pi_{P H S(P H S)}=\left(\frac{Q_{1}+Q_{2}}{2}\right) * p_{B}-\left(\frac{Q_{1}+Q_{2}}{2}\right) *\left[F+1-\phi\left(\frac{Q_{1}+Q_{2}}{2}+Q_{A O M}\right)\right]
$$

$$
\Pi_{M H N(P H S)}=\left[\lambda Q_{1}+\frac{(1-\lambda) Q_{1}+Q_{2}}{2}\right] * p_{B}-Q_{M i n} *\left(1-\phi Q_{M i n}\right)-F *\left(Q_{M i n}-\lambda Q_{1}\right)
$$

$$
-\left[\lambda Q_{1}+\frac{(1-\lambda) Q_{1}+Q_{2}}{2}-Q_{\text {Min }}\right] *\left[F+1-\phi\left(\lambda Q_{1}+\frac{(1-\lambda) Q_{1}+Q_{2}}{2}-Q_{\text {Min }}\right)\right]
$$

When switching from the pure hub and spoke network to the multi-hub network, the change to carrier A's profits is denoted by

(6) $\Pi_{M H N(P H S)}-\Pi_{P H S(P H S)}=\frac{1}{2}\left[\lambda Q_{1} * p_{B}-F * \lambda Q_{1}+\lambda Q_{1}\right]$

$$
-2 \phi\left[\lambda Q_{1} *\left[Q_{1}\left(1+\frac{1}{2} \lambda\right)+Q_{2}+Q_{A O M}\right]+2 Q_{\text {Min }} *\left[2 Q_{\text {Min }}-Q_{1}(1-\lambda)-Q_{2}-Q_{A O M}\right]\right]
$$

The first term represents the increase in revenues from the diverted demand, the savings from less feeding and the additional costs associated with transporting the additional passengers. The second term corresponds to the loss of density economics in the main hub as passengers are rerouted via the second hub. Due to the loss of density economics associated with multiple hubs, we would presume an airline employing such a network configuration to concentrate on a primary hub, while trying to minimize transfer traffic at the secondary hubs. This can be easily verified due to

$$
\operatorname{MC}\left(q_{1}\right)<M C\left(q_{2}\right) \text { when } q_{1}>q_{2}
$$

This implies that one hub always incurs lower costs than two hubs as given by

$$
\left(q_{1}+q_{2}\right) * M C\left(q_{1}+q_{2}\right)<q_{1} * M C\left(q_{1}\right)+q_{2} * M C\left(q_{2}\right)
$$

and that under the conditions of $q_{1}>q_{2}, q_{3}>q_{1}$ and $q_{4}<q_{2}$, it is always true that

$$
q_{3} * M C\left(q_{3}\right)+q_{4} * M C\left(q_{4}\right)<q_{1} * M C\left(q_{1}\right)+q_{2} * M C\left(q_{2}\right)
$$


essentially favoring one large and one small hub versus a setting with two hubs of equal size.

So while this has given some justification for the rationality of multi-hub networks beyond capacity restrictions, it cannot explain a strategy of equitable hubs. A possible reason is that we do not take into account airport costs. Therefore, the size of possible secondary hubs could be influenced by the degree of dependence between the airline and its primary hub airport. Airports - especially hub airports - have monopoly power, which often relates to higher costs. We would assume that the higher the costs for the hub airline at its primary hub, the higher the probability that it will follow a strategy of equitable hubs, as competition between the hubs leads to lower overall costs which can outweigh lower costs through density economics in concentrating traffic at one hub.

\section{$4 \quad$ Model discussion}

For both cases of the bypassed hub network and the multi hub network, the impact of changes of variables within the feasible parameter space are rather obvious. Operating a pure hub and spoke network becomes more advantageous with high density economics, low costs of feeding and a low preference of premium demand for direct flights. Conversely, initiating an additional non-hub long haul operation becomes attractive at low density economics, high costs of feeding and a high preference for direct flights. Elevated price levels speak in favour of the bypassed hub network, while high levels of demand tip the scale back towards a pure hub and spoke system as density economies can increasingly be realized.

The model only redistributes existing demand within the network when a carrier employs a direct connection. One may argue that the direct connection constitutes a completely new product which can attract additional demand. New direct routes set-up by low cost carriers have stimulated demand, however, this always went along with deep price cuts. But there are also long term effects that can originate due to a direct air connection as it represents an amelioration of the local infrastructure. We have not considered this effect, but the increase in demand should favour the direct connection.

Concerning the impact of network demand $Q_{A O M}$, the issues raised by the model seem quite counterintuitive. In comparison to a smaller airline, a carrier with an extensive hub and spoke network will be less inclined to offer non-hub flights, as the marginal capacity costs of the transfer connections are lower. Common sense would expect a major carrier to more readily bypass its hub, as its larger network has already reached a critical mass for hub and spoke operations. We believe that this is influenced by two facts: firstly, we assume capacity to be continuous and secondly, do not account for opportunity costs from lost density economics in the hub. However, to some extent the statement is backed by reality. The smaller legacy carriers seem to be more inclined to offer non-hub operations: Alitalia offers long haul flights from Rome in addition to Milano, Swiss from Geneva in addition to Zurich, and SAS from Stockholm in addition to Copenhagen. Conversely, 
larger carriers like Air France, Lufthansa, KLM, and British Airways have been rather restrictive with opening up non-hub routes. ${ }^{11}$

The results from propositions 1 and 2 become stronger in settings with more market participants, as the amount of passengers diverted from competition increases. The monopoly case on the other hand is straightforward. Profit maximisation for the carrier becomes a cost minimisation problem which leads to the adoption of a pure hub and spoke network configuration.

When relaxing the assumption, that only one of the hub and spoke carriers can decide to offer a direct flight, the Nash equilibrium can result in a Prisoner's Dilemma.

Proposition 3: Assume that offering a direct connection increases the profitability of an airline's network, then a setting where both carriers can choose to operate direct flights can result in a prisoner's dilemma.

Instigating a direct long haul connection increases total network costs. Under propositions 1 and 2, these are compensated by the additional revenues obtained by the exclusive offering of a direct flight. When both carriers operate a direct connection, no differentiation remains and demand is equally distributed resulting in the profit function:

$$
\Pi_{B H N(B H N)}=\left(p * \frac{\lambda Q_{S D}}{n}\right)-\left(\frac{\lambda Q_{S D}}{n} * M C\left(\frac{\lambda Q_{S D}}{n}\right)\right)+\Pi_{H S C}\left(\frac{(1-\lambda) Q_{S D}}{n}\right)
$$

To complete our duopoly setting, we need to derive a carrier's profit function when the competitor is the sole operator of a direct connection:

$$
\Pi_{P H S(B H N)}=\Pi_{H S C}\left(\frac{(1-\lambda) Q_{S D}}{n}\right)
$$

Due to $\partial M C(q) / \partial q<0$, we can determine

$$
\Pi_{P H S(B H N)}<\Pi_{B H N(B H N)}<\Pi_{P H S(P H S)}<\Pi_{B H N(P H S)} .
$$

which is illustrated as a numerical example in table 1 with $Q_{A O M}=100, Q_{S D}=50$, $\phi=0.005, p=3, \lambda=0.3, n=2, F=0.4$

\footnotetext{
${ }^{11}$ Ironically, Swiss, Alitalia, and SAS all have been in situations of financial distress. A possible reason for this situation is that the competitors Lufthansa, British Airways, and Air France rely on far larger feeding networks, while we only consider hub and spoke carriers with equal network size.
} 
Airline profit matrix

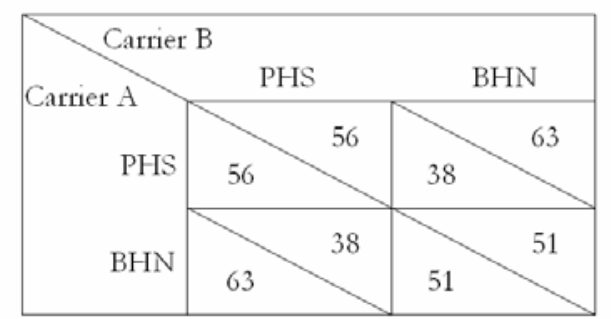

\section{Table 1}

It is therefore a strictly dominating strategy for a carrier to deploy capacity on the non-hub route. As profits in this case are below those obtained with pure hub and spoke network configurations, this constitutes a classic Prisoner's Dilemma. The outcome is analogous, when considering the case of a multi-hub network.

\section{$5 \quad$ Concluding remarks}

In short, we come to the following conclusions. Simple consideration of the cost side is not sufficient when evaluating network configurations. In a competitive market where the offering of a superior non-hub direct product can lead to a gain in high yield demand market share, cases exist where a profit maximising hub and spoke carrier will deviate from the polar case of a pure hub and spoke network. If demand on the non-hub route is not sufficient to enable operations, it can be rational for the carrier to operate a multi-hub network.

In the introduction, we have set out the question whether the on-going consolidation process in the European airline industry will have detrimental effects on the long haul services of secondary hubs. Exogenous restrictions currently impede any post-merger dehubbing of Amsterdam and Zurich, the hub cities of the two smaller merging partners KLM and Swiss. In the long term, the outlook remains ambiguous. Within the framework of our model, we expect long haul capacity outside the major hubs to decrease. We have asserted that it can be profit maximising for a hub and spoke carrier to operate non-hub long haul routes in addition to its hub and spoke network. An innovation of this paper is to offer an explanation for the presence of multi-hub networks beyond capacity restrictions the desire to seize a lucrative high yield market with a direct connection, while needing to satisfy a minimum of demand to operate aircraft profitably.

Hence, there will be situations where long haul connections will be continued, but we would expect the scale of operations to be lower than before. Should a city lose its hub status, capacity will be adapted to local demand needs. Even in the case of a multi-hubbing scenario, as put forward by proposition 2, our model suggests that the airline limits transfer traffic at the secondary hubs to minimize the loss of density economics. The affected cities will suffer economic consequences as direct employment associated with airline operations descends, but maintaining long haul routes for high yield demand will ensure the attractiveness for businesses. Likewise, supposed demand growth will allow more city pair markets to become economically interesting for direct connections. Inauguration of new 
direct flights, in turn, is suspected to lead to multiplier effects for the local economy. ${ }^{12}$ In some cases this will constitute a typical chicken and egg problem ${ }^{13}$, prompting local authorities to offer incentives for route development. ${ }^{14}$ Even if we do not expect these aids to degenerate in a similar manner as low cost carrier subsidises at quaternary airports, regulators should assure no distortion of competition occurs.

Proposition 3 poses some interesting implications for a post-consolidation era in Europe. Maintaining the long haul routes operated by the smaller merging partner is a rational approach when being able to rely on bilateral traffic rights that keep the local European competition at bay. Perhaps that is the rationality for European legacy carriers to reduce speed and scope on Open Skies talks between Europe and North America. Should long haul traffic be fully liberalized in the future, we may see the major hub and spoke carriers collude by retrenching around their hubs in an attempt to maximise profits. Nevertheless, this would not negate the case of a new entrant choosing to only operate direct routes. Similarly, retarding liberalization does not provide protection from carriers initiating direct flights from the long haul end. The US legacy carriers, under the increasing pressure on the domestic front, are turning to new transatlantic services. Delta is connecting New York with Hamburg and Berlin and Continental is starting to service routes from Newark to Hamburg, Berlin, and Stockholm. With proposition 4, we have given an explanation for the existence of multi-hub networks beyond capacity restrictions: the desire to seize a lucrative high yield market with a direct connection, while needing to satisfy a minimum of demand to operate aircraft profitably. Our model cannot explain the reasoning behind a strategy of equitable hubs but we have given a possible incentive: high dependency and costs at the airline's primary hub. Investigating these remaining open issues should be subject of further research.

\section{$6 \quad$ References}

Berechman, J. and Shy, O. (1996) "Airline Deregulation and the Choice of Networks," in Nijkamp, P. (ed), Recent Advances in Spatial Equilibrium Modelling. Springer, Berlin: 138-156.

Brueckner, J. and Zhang, Y. (1999) "Scheduling Decisions in an Airline Network: A Huband-Spoke System's Effect on Flight Frequency, Fares and Welfare," Working Paper 99/0110, Office of Research, University of Illinois at Urbana-Champaign.

Duedden, J. (2005) "Consolidation and its Implications for Direct Long Haul Travel," unpublished working paper, presented at the ATRS Conference 2005, Rio de Janeiro.

Hendricks, K., Piccione, M., and Tan, G. (1995) "The Economics of Hubs: The Case of Monopoly," Review of Economic Studies, 62: 217-230.

\footnotetext{
12 For example, Berlin is expecting positive economic impact from increased tourism and higher exposure for exhibitions from its new long haul routes.

${ }^{13}$ No direct operations as premium demand is too low, no premium demand as there are no direct operations.

${ }^{14}$ For the new New York-Berlin routes, hotels, tourism and city authorities have agreed to finance the travel costs incurred by Delta's and Continental's flight crews.
} 
Oum, T., Zhang, A., and Zhang, Y., 1995, “Airline Network Rivalry,” Canadian Journal of Economics, 28: 836-857.

Wojahn, O. (2001) "Airline Network Structure and the Gravity Model," Transportation Research Part E: Logistics and Transportation Review, 37: 267-279.

Wojahn, O. (2001) "Airline Networks if Passengers Value Time and Service Quality," unpublished working paper.

\section{$7 \quad$ Appendix}

In our model, we assume prices and quantities to be exogenously given, which constitutes a rather severe restriction. We impose this as we are interested in the impact of market share increases, rather than the effect of higher reservation prices to compensate for the additional costs incurred.

Under a setting with Bertrand competition, we would receive similar results. In the case where the carriers operate pure hub and spoke networks, both would price at marginal cost and make profits of zero. However, airline network carriers can and do price discriminate, so we can insinuate that $p$ is the average price of a range from $p_{H i}$ to $p_{\text {Low }}$ with $p_{H i}>p_{\text {Low }}$. As the weighted average price achieved equals marginal cost, we can derive that both carriers make a profit with high yield demand $\left(p_{H i}>M C\right)$ and a loss with low yield demand $\left(p_{\text {Low }}<M C\right) .{ }^{15}$

Proposition 3 becomes irrelevant, as the symmetry of both carriers will always lead to zero profits when choosing the same network configuration. Proposition 1 supposes that the rise in revenues due to the increase in market share can be sufficient to compensate higher costs. Under a Bertrand framework, the price dispersion can have a similar effect. When the increase in high yield market share can cover the higher costs of the direct operation, a carrier will be inclined to divert from its original network decision. Serving non-stop demand would allow the carrier to make profits, while pricing at marginal cost for the transfer passengers still yields zero profits.

Due to the loss of density economics, $p_{\text {Low }}$ increases when compared to the original scenario. In our model, this loss in consumer welfare declining profit margins for transfer passengers and is internalized by the carriers.

An alternative would be to associate the superior product of non-stop travel with a higher reservation price. Most of the issues above would still hold. It is rather straightforward that the decision to operate a direct flight then additionally depends on the premium that can be charged. When reaching a level sufficing to compensate the additional costs of the direct operation, a pure hub and spoke network configuration becomes suboptimal, even for the extreme cases of monopoly and $\lambda=0$. Again, proposition 3 must not hold anymore, as both carriers can increase their profits by offering additional non-hub connections.

\footnotetext{
${ }^{15}$ Airline prices are known to exhibit a high price dispersion between the lowest and highest price available. The lower end must not always cover cost, for example, free tickets by Ryanair. To secure profitable operations, the carrier needs to carry enough high yield passengers to compensate these losses.
} 
Concluding, deviating from our model and assuming Bertrand competition does not change much from the carrier's perspectives, while integrating a higher reservation price favours direct operations. Considering total welfare effects, we have to acknowledge the loss in consumer welfare as prices for low yield demand rise in the case of a non-hub operation. 